\title{
Representation of Vehicle Dynamics in Haptic Teleoperation of Aerial Robots
}

\author{
Xiaolei Hou, Robert Mahony, Felix Schill
}

\begin{abstract}
This paper considers the question providing effective feedback of vehicle dynamic forces to a pilot in haptic teleoperation of aerial robots. We claim that the usual state-ofthe-art haptic interface, based on research motivated by robotic manipulator slaves and virtual haptic environments, does a poor job of reflecting dynamic forces of a mobile robotic vehicle to the user. This leads us to propose a novel new force feedback user interface for mobile robotic vehicles with dynamics. An analysis of the closed-loop force-displacement transfer functions experienced by the master joystick for the classical and the new approach clearly indicate the advantages of proposed formulation. Both the classical and the proposed approach have been implemented in the teleoperation of a quadrotor vehicle and we present quantitative and cognitive performance data from a user study that corroborates the expected performance advantages.
\end{abstract}

\section{INTRODUCTION}

Force feedback or haptic teleoperation of remote robotic devices is a classical topic in robotics. The benefit of haptic feedback in teleoperation applications that require precision and skill of the user is well established [18], [4], [14], [9]. The utility of forcefeedback in teleoperation of mobile vehicles is less well studied. Obstacle avoidance and trajectory guidance control algorithms have been studied for terrestrial wheeled vehicles [5], [6] with force feedback generated by artificial force fields, typically virtual potentials or springdamper models associated with environmental interaction, that provides the user with a haptic sense of the local environment. In 2002 Lee et al [13] provided a user study that indicated that haptic feedback made a significant difference in the performance of a user in navigating through a complex obstacle strewn environment. The problem of force feedback teleoperation of a helicopter has been studied in the Faculty of Aerospace Engineering at Delft University of Technology over the last six years [2], [10], [12], [11]. This work has showed that a simple virtual potential or spring damper system does not provide good haptic cues to the pilot for obstacle avoidance. Instead they use ideas based on the generalized potential field [8] that compares estimates of time-to-contact and maximum stopping time to produce a force that becomes noticeable only when the vehicle is performing a manoeuvre that may lead it to come close to collision. Recent work by Brandt [3] also finds a similar time-to-contact cue to be the key to providing good haptic feedback to the pilot. Work by the Mahony [15],

Xiaolei Hou and Robert Mahony are with Research School of Engineering, Australian National University, Canberra, ACT 0200, Australia xiaolei.hou, robert.mahony@anu.edu.au

Felix Schill is with Laboratory of Intelligent systems, EPFL, Lausanne CH-1015, Switzerland. felix.schillepfl.ch
[20] has used optic flow as a direct cue for teleoperation of terrestrial wheeled vehicles and for aerial vehicles. The spherical divergence of an optical flow field for a moving vehicle is closely related to time-to-contact and the behavior of the system is qualitatively the same as that obtained in [2], [10], [3], [12], [11] with the added advantage that it is derived from a vision system, one of the lightest, most robust, exteroceptive sensor systems available for a aerial robot. Although there are a range of works concerning haptic rendering of exogenous forces to aid obstacle avoidance and task performance for aerial robotic vehicles the authors are aware of no work based on rendering the inertial forces of the vehicle.

In this paper, we propose a novel haptic control scheme that offers better representation of the dynamic force of mobile robotic vehicles in haptic teleoperation with a particular focus on aerial vehicles. The proposed approach is achieved by configuring the haptic joystick to servo control its position reference based on the velocity feedback from slave robot and measure force applied by user as the control reference input to the robot. This approach should be compared to the classical approach in which the position of the master joystick is used as input to the robot and the force applied to the joystick is derived from data received from the slave vehicle. The velocity of the vehicle is estimated by velocity observer using absolute position and attitude measurement from VICON visual tracking system to regulate the position set point of the joystick, providing the pilot with a feel for the motion of the vehicle, and to provides velocity controller with velocity feedback. The force applied to the joystick is measured and used as the velocity set point for the velocity controller of the aerial robot. In this way the pilot 'feels' the force applied to and the motion of the vehicle in a natural manner. Initial analysis indicates that the resulting controllability of the vehicle is significantly enhanced, and pilots have a much better perception of vehicle's motion and dynamics. A full factorial user study was carried out on a robotic experimental platform to verify that the proposed haptic interface performs significantly better than the stateof-art approach in both quantitative and cognitive measures.

The remainder part of this paper is organized as follows. Section II describes the problem formulation along with a comparison of the state-of-art approach for haptic teleoperation of mobile robots to the new proposed interface with an analysis of user perception of motion for each case. The approach we proposed to represent the vehicle's dynamics and comparisons upon user perception of two approaches are given in III. The results from the full factorial user 
